Historic, Archive Document

Do not assume content reflects current scientific knowledge, policies, or practices. 



\section{- PQDNIES 3-5 EYES.}

(CONTINUED)

La LORRAine. gLOBULAR CREAMy WHITE

LA PERLE, LILAC WHITE

LA TENDRESSE, MILK WHITE

La TUlipe, LILAC WHITE, LATE MIDSEASON

LIVINGSTONE, SOFT PINK, BEAUTIFUL

LORD KITCHENER, VERY FINE RED

Madame Calot, pinkish white, tinted flesh

Madame Emile Galle, sea shell pink

Madame Emile Lemoine. very large tall white

Madame GeISSLER. ROSE PINK

Madame Jules Dessert. fine blush white

Madame Mechin, deEp Red

Mademoiselle Rousseau. White, touched pink

Marcelle Dessert. Milk white, splashed lilac

Marguerite Gerard, hydrangea pINK

Marie Crousse, SOfT PINK, VERy LARge

MARIE JACQUIN. WATER LILY FORM. GLOSSY WHITE

MARQuis C, Lagergreen. CHERRY RED

MARY BRAND, LARGE CRIMSON RED

MIKADO. SINGLE CRIMSON AND GOLD

Monsieur Chas. Levecque, delicate pink

Monsieur Martin Cahuzac, very dark red

Monsieur Jules Elie. Large LILAC PINK

Monsieur Krelage. Currant red

Octavie Demay, del icate hydrangea pink

OFFICINALIS ROSEa, VERY EARLY PINK

Officinalis Rubra Plena, old fashioned Red

OfFICINALIS TENUIFOLIA, DOUbLE RED fRINGED LEAVES

PASTEUR, SOFT PINK

PIERRE DESSERT, DARK CRIMSON

PIERRE DUCHARTRE. LILAC PINK

PRESIDENT TAFT. LARGE PINK. SUPERB

PRIMEVERE, LARGE SULPHUR YELLOW

QUEEN VICTORIA, A GOOD POPULAR WHITE

QUEEN WILHELMINA, ROSY PINK, VERY LARGE

RI CHARD CARVEL, BEST EARLY RED

RACHEL. (LEMOINE) CLEAR PINK

Reine Hortense, (President taft)

RUBRA SUPERBA, RICH DEEP RED

SARAH BERNHARDT. MAUVE ROSE

SOLANGE, WAXY WHITE

SuzetTe, Bengal rose

THERESE. LARGE SATINY PINK

TOURANGELLE, PEARLY WHITE

WALTER FAXON, BRIGHT ROSE PINK

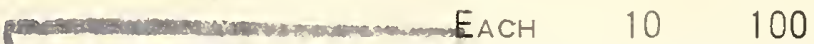

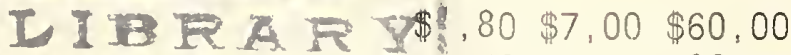

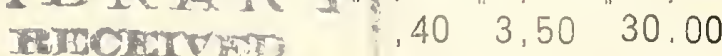

AUG $231030+\quad 50 \quad 4,00 \quad 30,00$

$.50 \quad 3,00 \quad 25,00$

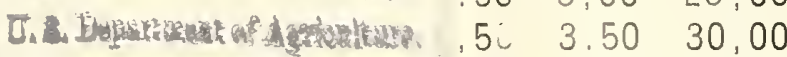

$.80 \quad 7,00 \quad 55,00$

$, 30 \quad 2.50 \quad 20,00$

$.40 \cdot 3,00 \quad 25,00$

$.60 \quad 5.00 \quad 45.00$

$50 \quad 4,00 \quad 35.00$

$2.50 \quad 12,50 \quad 100,00$

$.50 \quad 4.00 \quad 35.00$

$80 \quad 7,00 \quad 60.00$

$\begin{array}{lll}60 & 5.00 & 40.00\end{array}$

$\begin{array}{lll}40 & 3.00 & 25.00\end{array}$

$80 \quad 7.00 \quad 55,00$

$, 40 \quad 3.50 \quad 30,00$

$.80 \quad 7.00 \quad 55.00$

$1.20 \quad 8.00 \quad 75,00$

$1.20 \quad 10.00 \quad 90,00$

$\begin{array}{lll}50 & 3.50 & 30.00\end{array}$

$2.00 \quad 10.02 \quad 8.00$

$\begin{array}{lll}.65 & 5.00 \quad 45.00\end{array}$

$\begin{array}{lll}50 & 3,00 & 25,00\end{array}$

$60 \div \sim 35.00$

$\begin{array}{lll}65 & 5,00 & 40.00\end{array}$

$65 \quad 4,50 \quad 40,00$

$1,50 \quad 12,00 \quad 100,00$

$\begin{array}{lll}1,00 & 9,00 & 75,00\end{array}$

$, 60 \quad 5,00 \quad 4.0 .00$

$80 \quad 7.00 \quad 55,00$

$.75 \quad 6,00 \quad 45,00$

$1,50 \quad 7.50$

$.30 \quad 2.50 \quad 18,00$

$\begin{array}{lll}1.00 & 9.00 \quad 75.00\end{array}$

$2.50 \quad 15.00$

$\begin{array}{lll}.75 & 6.00 & 45.00\end{array}$

$\begin{array}{lll}75 & 4.50 & 40.00\end{array}$

$\begin{array}{lll}50 & 3.50 & 30.00\end{array}$

$, 90 \quad 5,50 \quad 50,00$

$2,00 \quad 10,00$

$.504 .00 \quad 35.0^{7}$

$2.5 ; 10,0$ )

2. $, 0 \quad 18,00 \quad 150,00$

$2,50 \quad 12,50$

PEONIES TO COLOR

WHITE VARIETIES MIXED

PINK VARIETIES MIXED

RED VARIETIES MIXED
$\$ 100.00$ PER 1000

$\$ 100.00$ PER 1000

$\$ 150.00$ PER 100 J $\begin{array}{lll}.25 & 2.00 \quad 15.00\end{array}$

$\begin{array}{lll}.25 & 2.00 \quad 15.00\end{array}$

$\begin{array}{lll}30 & 2.50 & 18.00\end{array}$ 


\section{WGH QUALITY ASSOHTMENT OF IRIS}

\begin{tabular}{|c|c|c|}
\hline \multicolumn{3}{|c|}{ PER 100} \\
\hline AFTERGLOW. LAVENDER & $\$ 10,00$ & LOHENGR IN, PINK \\
\hline ALCAZER, LILAC BLUE & 8,00 & LORD OF JUNE, VIOLET BLUE \\
\hline AMBASSADOR, PURPLE & 15.00 & Mother of PEaRL \\
\hline ANNA FARR, WHITE AND BLUE & 25.00 & ORIFLAMME, RICH PURPLE \\
\hline AUREA. YELLOW & 8,00 & PALLIDA DELMATICA, FINEST OF ALL \\
\hline Black Prince, deEP blue & 6.00 & PALLIDA SPECIOSA, LAVENDER \\
\hline CAPRICE & 8.00 & PURPLE KING, PURPLE \\
\hline Cecile Minturn. pink & 10.00 & Perladonna, China blue \\
\hline DOROTHEA, SOFT BLUE & 8.00 & PrINCESS BeATRICE \\
\hline DALILA & 8.00 & QueEn Caterina, LaVender \\
\hline DEJAZET & 10,00 & QUEEN OF MAY. PINK \\
\hline DUKE OF YORK & 20.00 & RHEIN NIXIE, BLUE \\
\hline EMPEROR (SIB.) & 12.00 & ROSE UNIQUE \\
\hline HER MAJESTY, FINE PINK & 7.00 & SEMINOLE, CRIMSON \\
\hline IRIS KING. OLD GOLD & 9.00 & SNOW QUEEN $(S$ IB. $)$ \\
\hline JEANNE dE ARC, NEW WHITE & 8.05 & SUNNYBROOK FARM $\left(S_{I B,}\right)$ \\
\hline LAVENDER, FINE LAVENDER & 6.00 & SHERWIN WRIGHT, YELLOW \\
\hline LeVERrier. ChINese VIOLET & 40.00 & Trojana Magnifica. \\
\hline LENT A. WILLIAMSON, EARLY & 17,00 & \\
\hline
\end{tabular}

Per 100

$\$ 8,00$

25,00

25,00

8,00

6,00

7,00

8,00

10.00

12.00

15.00

5.00

5,00

8.00

8.00

8.00

10.00

5.00

8.00

\section{PEONIES \\ NEW AND CHOICE VARIETIES \\ 3-5 EYES.}

Albatre, MILK WHite

Albert Crousse, Sea SHell pink

Alsace -LoRraine, creamy WhIte

AUGUSTIN D'HOUR, LARGE BRILLIANT RED

AVALANCHE, MILK WHITE

BARONESS SCHROEDER, VERY FINE WHITE

BOULE DE NEIGE, MILK WHITE

Claire Dubois. deep rose pink large flowers

COURONNE D'OR, WHITE YELLOW CENTER

DR: H. VANDER TAK, Claret RED

Duchesse de Nemours, SUlphur White

EDOUARD ANDRE, DARK VELVETY CRIMSON

EDULUS SUPERBA. POPULAR PINK

EMILE Hoste, Very large creamy whIte, fine

EUGENIE VERDIER, TALL LARGE PINK

Felix Crousse, popular Red

Festiva Maxima, splendid large White

GeORgIanA SHAYLOR, LARge FLESH PINK

GERMAINE BIGOT, LILAC ROSE

GIGANTEA, LARGE PINK

GISMONDA, VERY LATE, FLESH PINK

Grover Cleveland; DARK RED

HuMEI, CLEAR FLESH, TINGED ROSE

JOHN HANCOCK. TALL CRIMSON

Karl Rosenfield. Best COMmercial Red

La France, BEAUTIFUL LARgE PINK
EACH $10 \quad 100$

$\$ .50 \$ 3.00 \$ 25.00$

$.50 \quad 3,50 \quad 30.00$

$.80 \quad 8.50 \quad 80.00$

$.60 \quad 5.00 \quad 40,00$

$.50 \quad 2.50 \quad 20.00$

$, 70 \quad 5,50 \quad 50,00$

$\begin{array}{lll}.45 & 2.50 \quad 20,00\end{array}$

$.60 \quad 4.00 \quad 35,00$

$.35 \quad 2.50 \quad 20,00$

$.80 \quad 7,00 \quad 60,00$

$.30 \quad 2,20 \quad 18,00$

$, 60 \quad 3,00 \quad 25,00$

$, 30 \quad 2.20 \quad 18,00$

$\begin{array}{lll}.70 & 6.00 & 45.00\end{array}$

$.45 \quad 3,50$

$\begin{array}{rrr}, 50 & 4,00 \quad 35.00\end{array}$

$.30 \quad 2.20 \quad 18,00$

$2,50 \quad 10.00$

$.40 \quad 3.00 \quad 20.00$

$.60 \quad 5.00 \quad 40.00$

$.60 \quad 5.00 \quad 40.00$

$\begin{array}{lll}.70 & 4,50 \quad 40,00\end{array}$

3) $2,50 \quad 18,00$

$625 . \cong 0 \quad 40.00$

$.70 \quad 4.00 \quad 35.00$

$3,00 \quad 15.00 \quad 125,00$ 
PER 1000

SINGLE LATE OR COTTAGE TULIPS,

BOUTON D'OR. GOLDEN YELLOW

ELLEN WILLMOTT. SOFT YELLOW

FAIRY QUEEN, HELIOTROPE

Gesneriana Spathulata, scarlet

Gesneriana Macrospella, Red

GESNERIANA, IXIOIDES, YELLOW

INGLESCOMBE YELLOW

I NGLESCOMBE PINK

INGLESCOMBE SCARLET

I SABELLA ROSY WHITE

MRS, MOON, PURE YELLOW

PARISIAN WHITE

PARISIAN YELLOW

PARISIAN SCARLET

PICOTEE, PINK EDGE

CHOICE MIXED ALL COLORS

BREEDER TULIPS,

BACCHUS, DARK BLUE

BRONZE QUEEN

Cardinal Manning, rose

FEU ARDENT, RED FORCING

Godet Parfait, PURple-Violet
$\$ 24,00$

20.00

24,00

24.00

20,00

32,00

24,00

20,00

20.00

20,00

40,00

20,00

26.00

20.00

24.00

20,00
BREEDER TULIPS

JAUNE D'OEUF, BRONZE

LOUIS XIV, DARK PURPLE

Panorama, RED

Prince of Orange

St, James, DARK PURPLE

VELVET KING, PURPLE

Yellow PERfECTION. Yellow

CHOICE MIXTURE ALL COLORS

LILIUM RUBRUM

8-9 IN. 225 TO CASE

9-11 IN, 180 TO CASE

LILIUM AURATUM

$8=9^{*} 225$ TO CASE

9-11" 180 to CASE

60,00

30,00

40,00

50,00

50,00
LILIUM Regale 5-6"

LILIUM TIGRINUM SINGLE

LILIUM TIGRINUM DOUBLE

LILIUM ELEGANS

LILIUM UMBELLATUM

PER 1000

$\$ 30,00$

50,00

30.00

50.00

50.00

50.0

50.00

25.00

PER CASE

25.00

25,00

Per case

20.00

20.00

PER 1000

150,00

150,00

150,00

150,00

200,00

\section{CHOICE NEW VARIETIRS OF IRIS}

Apache

Alabaster

ASIA, PURPLE AND RED

APHRODITE, VIOLET-PINK

BERTRAND

Citronella. Golden yellow

Coppersmith. INDIAN LAKE

DOROTHEA K. WILLIAMSON

DUKE OF BEDFORD

ELAINE

FRIEDA MOHR. PINKISH LILAC

Gold ImPERIAL. Chrome yellow

GEORge J. TriboLet

I DUNA

JUBILEE, PEACH COLOR

KINGFISHER BLUE $\left(S_{I B},\right)$

LONDON

LEVERRIER, LIGHT RED

MOROCA IN

Monsieur Arnal. REDdish Brown

MAJESTIC, LIGHT LAVENDER
$\mathrm{EACH}$

$\$ 2,00$

1.25

1,00

2,00

1,00

.80

3,50

.50

2.25

1,75

1.75

1.00

2.25

1.00

1,75

.40

1.00

.40

.40

2.00

.80
Marjorie Tinley, blue

Michelline ChairRaire. White

MILDRED PRESBY, WHITE

Mme, Henrie Cayeaux

Morning SPLENDOR, VIOLET

MRS. MARION CRAN, PINK

MIDGARD, YELLOW AND ROSE

MOONLIGHT, FRENCH GRAY

PEERLESS, RED

PIONEER, RED-PURPLE

ROMOLA, PALE LILAC

R. W, Wallace, Rich Violet

SNOW WHITE

Souv, de MME, GaUdichar

SIMMONE VASSIERE, WHITE

$\mathrm{EACH}$

$\$ .80$

2.75

.80

2,30

1.00

2.00

5.00

1.00

7.25

2.00

4,25

3,50

3,50

, 50

, 80

3.00

1,00

.80

2.00

.80

3.00 
THESE PRICES INCLUde FREIGHT AND DUTy PAID to ChICAgo,

\section{HYACINTHS}

EXHIBITION SIZE 19 CTMS UP

FIRST SIZE 18-19 CTMS

SECOND SIZE $17 \cdot 18$ CTMS

THIRD SIZE 16-17 CTMS

FORCING SIZE 15-16 CTMS

BEDDING SIZE 14-15 CTMS

MINIATURE SIZE 13\%14 CTMS

WE SUPPLY THE FOLLOWING VARIETIES:

L' Innocence, La Grandesse, Arentine

Arendsen. Queen of Whites. Gertrude,

Lady Derby, La Victorire. Queen of

PINKS, BISMARCK. GRand MaITRE. King of

Blues, Queen of Blues. Perle Brilliant.

Yellow Hammer. City of Harlem and Mixed. WHICH ARE SOLD IN 100 LOTS.

\section{PREPARED HYACINTHS}

L'I NNOCENCE, VICTOR EMANuel,

LA VICTORIRE, BISMARCK, FORCING SIZE

15-16 CTMS PER $1000 \$ 160,00$

\section{SINGLE EARLY TULIPS}

ARTUS SCARLET RED

20,00

BRILLIANT STAR, RED FORCER

Couleur Cardinal, CRIMSON

CRAMOISI Brilliant. RED

DIANA, PURE WHITE

FLAMINGO, ROSE-PINK

LA REINE, BLUSH WHITE

La Reine Maxima, light pink

L'EsPERANCE, MAUVE PURPLE

MON TRESOR, YELLOW EARLY

Prince of AUSTRIA RED

36,00

32,00

20.00

20,00

32,00

20,00

28,00

32,00

44.00

34.00

40,00

Proserpine, VIOLET-ROSE FORCER

PPOSPERITY, DEEP PINK EARLY FORCER

ROSE GRIS.DE-LIN. LIGHT PINK

ROSE LA REINE, BRIGHT PINK

ROSE LUISANTE, BRIGHT ROSE

Rose PRecoce, EARLIEST PINK

VERMILLON BRILLIANT; RED

WAPEN VAN LEIDEN PINK

WHITE HAWK EARLY FORCER

YELLOW PRINCE, YELLOW FORCER

Choice Mixture all colors

\section{DOUBLE EARLY TULIPS}

Boule de NEIGE WHITE

CouronNe D'OR YeLLOW

EL TOREAdOR, RED ORANGE

24,00

30,00

40,00

44,00

32,00

40.00

32.00

40,00

20,00

30.00

36.00

50,00

\section{DOUBLE EARLY TULIPS}

MURR ILLO, PINK FORCER

MR. VAN DER HOEF, YELLOW

L'AURORA, ORANGE RED

PEACH Blossom PINK FORGer

Rubra Maxima Red

SALVATOR ROSA DEEP ROSE

SCHOONOORD. WHITE

TEA ROSE. YELLOW

TOURNESOL, RED YELLOW

VUURBAAK, RED FORCER

Choice Mixture all COLORS

DARWIN TULIPS

Allard Pierson, Christmas forcer

Adolphe Van der HeEde red

70,00

ANTON ROOSEN PINK

BARRONNE DE LA TONNAYE ROSE

BARTIGON, EARLY RED

CENTENAIRE, LILAC=PINK

Clara Butt, salmon pink

DREAM, DEEP LILAC

FARNCOMBE SANDERS, SCARLET

FEU BRILLIANT, SCARLET

GRETCHEN, SOFT PINK

Gustave DORE, PINK

HOMER, EARLY SCARLET

HERODIADE, PINK FORCER

LA TUlipe NOIRE. BLACK

LENOTRE ROSE-PINK

LOVELINESS, SOFT ROSE

MME, Krelage, ROSE-PINK

PaINTEd Lady, GREAM WHITE

PSYCHE, LIGHT ROSE

Pride of HaArlem. Amer, Beauty

PRINCESS ELIZABETH, VIVID PINK

PHILIPPE DE COMINES. MAROON-BLACK

Prof, Rauwenhof, light red

REV. EWBank. HELIOTROPE

ROI D'I SLANDE. CARMINE

SIERAAD VAN FLORA. ROSE=PINK

THE SULTAN. BROWN-BLACK

White QueEn. LARge WHITE

WM. COPLAND. EARLY FORCER
WILLIAM PITT, EARLY FORCER

ZULU, VIOLET-BLACK

Cholce Mixture, All colors
PER 1000

$\$ 24,0$

44.00

40,00

40.00

30.00

40.00

30.00

28,00

36.00

36,00

24,00
32,00

32,00

24,00

24,00

26.00

24,00

28,00

24, 00

30,00

24,00

24,00

24,00

30.00

30,00

26.00

24.00

24.00

24.00

24. 00

24.00

28.00

24.00

26.00

26.00

30,00

24,00

24.00

26.00

30.00

30.00

26.00

20.00 